\title{
Trivium
}

Revue franco-allemande de sciences humaines et sociales - Deutsch-französische Zeitschrift für Geistesund Sozialwissenschaften

1 | 2008

"Iconic Turn » et réflexion sociétale

\section{Die Linie der Ehelosigkeit}

Der Zufall, die Arabeske und die Volute: Beitrag zu einer Geschichte des Zigloogloo

\section{Didier Semin}

Traducteur : Annette Leppert-Fögen

\section{(2) OpenEdition}

\section{Journals}

Édition électronique

URL : http://journals.openedition.org/trivium/243

DOI : $10.4000 /$ trivium.243

ISSN : 1963-1820

Éditeur

Les éditions de la Maison des sciences de l'Homme

Référence électronique

Didier Semin, « Die Linie der Ehelosigkeit », Trivium [Online], 1 | 2008, online erschienen am 08 April 2008, abgerufen am 08 September 2020. URL : http://journals.openedition.org/trivium/243 ; DOI : https://doi.org/10.4000/trivium.243

Ce document a été généré automatiquement le 8 septembre 2020.

\section{cc)}

Les contenus des la revue Trivium sont mis à disposition selon les termes de la Licence Creative Commons Attribution - Pas d'Utilisation Commerciale - Pas de Modification 4.0 International. 


\section{Die Linie der Ehelosigkeit}

Der Zufall, die Arabeske und die Volute: Beitrag zu einer Geschichte des Zigloogloo

Didier Semin

Traduction : Annette Leppert-Fögen

\section{NOTE DE L'ÉDITEUR}

Aus urheberrechtlichen Gründen ist es uns leider nicht möglich, alle im Originalartikel enthaltenen Abbildungen an dieser Stelle zu reproduzieren. Der interessierte Leser wird gebeten, die Originalausgabe heranzuziehen.

Ein besonderer Dank gilt Didier Semin für seine Unterstützung beim Finden geeigneter Reproduktionen und J. Monnier für die kostenlose Bereitstellung der Abbildung zu Marcel Duchamp. Dem Herausgeber des Originaltextes, den Cahiers du Musée national d'art moderne, sei an dieser Stelle ebenfalls ein Dank ausgesprochen für die freundliche Genehmigung zur Übersetzung.

\section{Zufallsmusik}




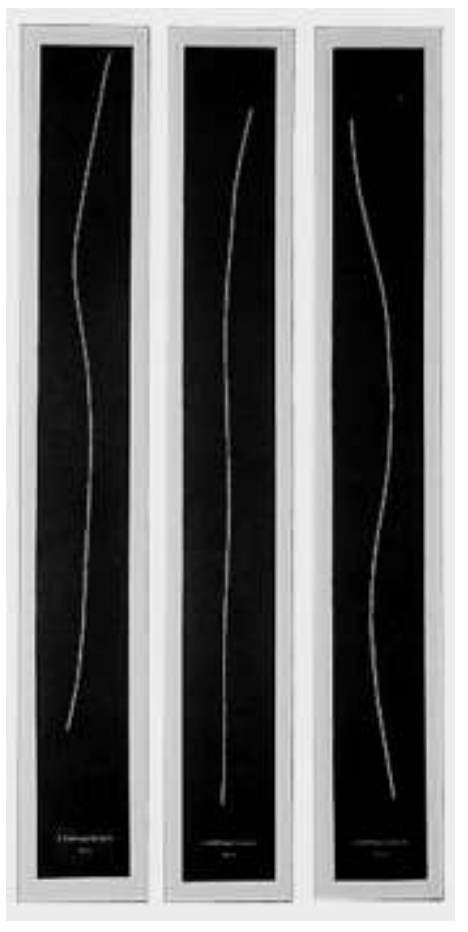

Drei Fäden auf drei mit Preußischblau bemalten Leinwandstreifen befestigt, auf Glas montiert. Kopie 1964. Sammlung des Musée national d'art moderne/CCI, Centre Georges Pompidou, Paris.

(c) ADAGP, J. Monnier

1 In Raymond Roussels Romanen ist die Zufallsmusik ein ständiges Motiv. Zu Beginn der Eindrücke aus Afrika spielt ein Mitglied des Clubs der Unvergleichlichen die Rolle von Georg Friedrich Händel, wie er auf einem Treppengeländer die Partitur eines Oratoriums schreibt, "das von einem vom Zufall gelieferten Verfahren mechanisch konstruiert worden sei«. ${ }^{1}$ Die Extravaganz dieses Verfahrens wird etwa hundert Seiten später erklärt: Eines Tages habe Händel sich gegen Ende eines Banketts dazu herausfordern lassen, eine Melodie nach dem Zufallsprinzip zu komponieren, und diese Leistung unverzüglich und ausschließlich mit Hilfe dessen vollbracht, was ihm gerade zur Hand war - einer Gänsefeder, einem Treppengeländer, den Zweigen einer Stechpalme und Seidenbändern. ${ }^{2}$ Nicht die Idee einer Zufallsmusik als solche ist im Jahre 1910 außergewöhnlich, hatten im 18. Jahrhundert doch zahlreiche Lehrbücher Nichtmusiker im Komponieren von Walzern und Menuetten mithilfe von Würfeln unterrichtet ${ }^{3}$ : Den Usus, ja sogar die Urheberschaft schrieb man den ganz Großen zu Haydn oder Mozart -, und als bewanderter Pianist hatte Roussel sicherlich davon erfahren. Eher ist es das Augenmerk, das er auf die Einzelheiten, die unglaublichen Raffinessen des vom Komponisten angewandten Verfahrens richtet, und der Umstand, dass er dem Entstehungsprozess letztlich mehr Bedeutung einräumt als dem Resultat, was Roussels Fiktion valérysche Modernität verleiht. Auch wenn »Händel, sein VesperOratorium mechanisch komponierend" sich nur über ein paar Seiten der Eindrücke erstreckt, darf man vermuten, dass Roussel selber sich hinter den Konturen des Komponisten verbirgt: Präsentiert er doch in Comment j'ai écrit certains de mes livres sein gesamtes literarisches Werk als ein gewaltiges Kryptogramm, hinter dem keine andere Wahrheit stecke als die des Prozesses der Entstehung jener Bücher - eines unendlich komplexen Prozesses. 
Die beiden von Marcel Duchamp Ende 1914 geschriebenen Partituren mit dem Titel Musical Erratum ${ }^{4}$ werden ebenfalls als vollkommen aleatorisch betrachtet, könnten sie doch, warum auch nicht, den Eindrücken aus Afrika manches verdanken, die Duchamp $1912 \mathrm{im}$ Theater gesehen hat - und wenn nicht der Szene des komponierenden Händel, so doch zumindest der des Ver de terre joueur de cithare (an die er sich in seinen Gesprächen mit Pierre Cabanne erinnert ${ }^{5}$, und die eine weitere Figur des musikalischen Zufalls bei Roussel darstellt). Beide Versionen des Musical Erratum sind ebenfalls bedeutender in dem, was sie über ihre Genese zum Ausdruck bringen, als in ihrer musikalischen Qualität: Die eine wird in der Grünen Schachtel neu aufgelegt, aber, nach allem, was man weiß, erst ein halbes Jahrhundert nach ihrer Komposition uraufgeführt werden $^{6}$, und dies nicht auf Duchamps Initiative. Unter der zweiten, erst spät entdeckten Version ist vermerkt, dass die Aufführungsdauer des Stücks variabel, vor allem aber, dass diese Aufführung "gänzlich unnütz« sei.7 Es handele sich um Partituren, die man zeigen oder erzählen kann, aber nicht um Partituren zum Spielen.

Der zeitgenössische Theoretiker der Aleatorik in der Musik, John Cage, ist unter diesem Gesichtspunkt nur auf paradoxe Weise Duchamps musikalischer Erbe, steht doch Cages Begeisterung für den Ton als physikalisches Phänomen sehr im Gegensatz zu der Gleichgültigkeit, die der Autor des Großen Glases hinsichtlich der Aufführung seiner Partitur manifestierte. Die Unbestimmtheit ist in Cages Ethik selbstverständlich essenziell. Und parallel zu den Regeln des IGing, von dem er sich meist die Zufallsoperationen diktieren ließ, bediente er sich hie und da subtiler Kniffe, deren Komplexität der Treppe des Händel aus den Eindrücken um nichts nachstand. Eins seiner Werke, HPSCHD $^{8}$, ist sogar von der Mozart zugeschriebenen Methode des Würfelspiels inspiriert, und wenn Cage sich in Nam June Paiks Good Morning, Mr. Orwell 9 bemüht, einer Feder und einem Kaktus Töne abzugewinnen, scheint er direkt der Fantasie Roussels entsprungen. Doch bleibt für Cage die Darbietung, die Aufführung der Schlüssel zu jeglicher Zusammenstellung von Tönen: »Die Zufallsoperationen«, deren Durchführung er sich »in disziplinierter Form « wünscht ${ }^{10}$, werden als ein an den Komponisten persönlich gerichteter moralischer Imperativ betrachtet und keineswegs als etwas, was ausschließlich als Kompositionstechnik eingesetzt wird. Cages Partituren, selbst jene von 4'33 aus dem Jahre 1959, die aus einem weißen Blatt Papier besteht, waren zum Spielen und zum Hören da und sind es geblieben.

\section{Die Kunst des Fallenlassens}

4 Von da her kann man verstehen, dass Cage für die 3 Maßnorm-Stoppagen - nach Marcel Duchamp sind sie "konservierter Zufall «11 - eine ganz besondere Faszination empfand und dass er nicht nur über ihr Warum reflektierte, sondern auch über ihr Wie - das heißt über die Einzelheiten des Verfahrens, das Duchamp empfohlen hatte, um ein aleatorisches Ergebnis zu erhalten.

5 Das Warum der Stoppagen ist in groben Zügen bekannt: Für Duchamp handelte es sich darum, »einen pataphysischen Zweifel am Konzept der geraden Linie als dem kürzesten Weg zwischen zwei Punkten aufsteigen « zu lassen. ${ }^{12}$ Kurz und gut, darum, der jungen Frau aus Sartres Ekel schon vorab Kummer zu bereiten, die doch so froh ist zu wissen, dass der Urmeter irgendwo als festes Element vorhanden ist ${ }^{13}$, und ihr beizubringen, in der ungewissen Welt der amüsanten Physik zu leben, in der zwar alles möglich, aber niemals sicher ist. 
6 Man glaubt, das Wie noch besser zu kennen. Eine Notiz zur Schachtel von 1914 weist unter der Überschrift »Idee der Fabrikation« auf die Hypothese des Verfahrens hin, die als Präludium zum Werk dient:

»Wenn ein gerader, horizontaler Faden von einem Meter Länge aus einem Meter Höhe auf eine horizontale Ebene herunterfällt, sich nach seinem Belieben verändert und eine neue Figur von der Längeneinheit ergibt - 3 Exemplare, unter nahezu ähnlichen Bedingungen erhalten [es folgt eine Parenthese von vier durchgestrichenen Zeilen]: in ihrer Betrachtung Jedes zu Jedem, sind eine angenäherte Rekonstitution der Längeneinheit. Die 3 Maßnorm-Stoppagen sind der verminderte Meter « $^{14}$;

die Eintragung auf der Rückseite der Stoppagen (»ein Meter gerader, horizontaler Faden, der aus einem Meter Höhe heruntergefallen ist (15) $^{15}$ scheint zu bestätigen, dass jene das Ergebnis der Aktion sind, die in der Notiz beschrieben ist. Und in seinen Ausführungen im Vortrag "Hinsichtlich meiner selbst $«{ }^{16}$ wie auch in denen gegenüber Richard Hamilton ${ }^{17}$ drückt Duchamp sich noch deutlicher aus: Die Fäden wurden in einer Höhe von einem Meter über in Preußisch-Blau gefärbter, auf ein Gestell gespannter Leinwand losgelassen; danach wurden sie mit Hilfe von Firnistropfen fixiert und genau so in der Lage fotografisch festgehalten, in der sie von dem unerwarteten Ende ihres Falls überrascht wurden.

7 Von den verformten Fäden wurden (wahrscheinlich im Jahre 1918 ${ }^{18}$ ) Holzmodelle angefertigt, so dass Duchamp ihre Linienführung genau ins Große Glas und in $D u$... mich übertragen konnte. 1936 wurden Leinwand und Fäden auf Glas gesetzt und mitsamt den Modellen in einer Holzkiste für Krocketspiele untergebracht.

8 So war es nur folgerichtig, dass John Cage eines Tages aus der Perspektive des Musikers daran dachte, die Partitur der Stoppagen zu spielen, was bedeutete, Duchamps Aktion zu reproduzieren und zu sehen, was dabei herauskommt. Zwar befolgte er peinlich genau die Anweisungen der Schachtel von 1914, erzielte jedoch nichts, was den Linien der Stoppagen irgendwie verwandt gewesen wäre ... Für ihn war in der Weigerung des Zufalls, sich auf Geheiß einzustellen, zweifellos eine Zen-Weisheit erkennbar. In jüngerer Zeit jedoch und in eher kartesianischer Perspektive hatten Rhonda Shearer und Stephan Jay Gould die Idee, den Prozess der Stoppagen erneut, und zwar seitens der experimentellen Wissenschaften, einer Überprüfung auszusetzen ${ }^{19}$, indem sie John Cages Versuche in methodischer Form wiederholten. Das Ergebnis ist überraschend: Trotz der Behauptungen Marcel Duchamps ${ }^{20}$ und obwohl es unter allen nur denkbaren Bedingungen und mit verschiedenen Fäden wiederholt wurde, scheint dieses Experiment zu belegen, dass ein Faden von einem Meter Länge, der über einer horizontalen Unterlage fallengelassen wird, sich nicht entsprechend dem Modell der Stoppagen verformt. Es ist nicht einfach nur so (und hiervon können Tom Tit's Nacheiferer sich überzeugen, indem sie die Operation zuhause wiederholen), dass die Linien, die man erhält, anders verlaufen als die der Stoppagen - in einem solchen Fall könnte man ja ohne weiteres einräumen, dass der Zufall nicht zweimal dieselbe Form erzeugt, nicht einmal am Ende einer sehr langen Versuchsreihe mit identischer Anordnung. Nein, der Linienverlauf in den Stoppagen gehört so offenkundig zu einer anderen Gattung als der, die sich einstellt, wenn man peinlich genau das vom Künstler beschriebene Procedere nachvollzieht - dass sich bezweifeln lässt, dass er dieses Verfahren überhaupt jemals eingesetzt hat. 


\section{Kräuselbänder}

9 Als Rhonda Shearer im MoMA die Stoppagen näher betrachtete, stellte sie obendrein fest, dass die drei Filamente, von denen man annahm, dass sie auf ihrer Unterlage aus blauer Leinwand klebten, dort in Wirklichkeit nicht nur aufgeklebt, sondern irgendwie aufgenäht waren ${ }^{21}$ : Sie ziehen sich jeweils vom einen Ende zum anderen über die Leinwand und ragen an der Rückseite um einige Zentimeter darüber hinaus, sind also länger als ein Meter. Und hieraus folgert Shearer, dass sie sich in einer solchen Anordnung für ein Arrangement geradezu anbieten - etwa so, wie man den Faden in jenen Spielen arrangiert, bei denen man "nach seinem Belieben" das Profil eines Menschen auf einer Pappe gestalten kann. Welche Einwände andere DuchampSpezialisten gegen Rhonda Shearers Betrachtungen auch geltend machen mögen (die Maßnorm-Stoppagen, die sich früher im Besitz von Katherine Dreier befanden, blieben lange Zeit in einer Möbelaufbewahrung mehr oder weniger sich selber überlassen, bevor der Künstler sie 1936 wieder an sich nahm, und es wäre doch geradezu typisch für ihn, wenn er den einfachsten Weg gewählt hätte, um ein Werk zu reproduzieren oder zu restaurieren), ihre Hypothese ist jedenfalls außerordentlich verlockend: dass nämlich Duchamp es mit dem Zufall nicht so genau genommen habe.

10 Wie sieht nun ein Faden von einem Meter Länge aus, der unter normalen atmosphärischen Bedingungen aus einem Meter Höhe heruntergefallen ist? Nach nichts, wird man spontan antworten, und dieses Nichts hätte Duchamp dazu verleiten können, etwas zu schummeln - wenn er es denn getan hat. Da aber die "Indifferenzschönheit «"22 genau das Thema ist, dem Duchamp sein Leben als Künstler gewidmet hat, wird man wohl eher von der umgekehrten Hypothese ausgehen müssen: Ein Faden von einem Meter Länge, der unter normalen atmosphärischen Bedingungen aus einem Meter Höhe heruntergefallen ist, könnte in Wirklichkeit sehr wohl Formen annehmen, die viel zu bildlich-anschaulich sind, als dass Duchamp sie als solche hätte akzeptieren können. Denn traditionell (von Plinius über Leonardo da Vinci bis zu Cozens) wurde der Zufall in der Malerei ja nicht deshalb bemüht, weil er eventuell ganz neue Formen hätte entstehen lassen können, sondern wegen seiner Fähigkeit, Bilder hervorzurufen, die Ähnlichkeit mit der bekannten Realität besitzen. 


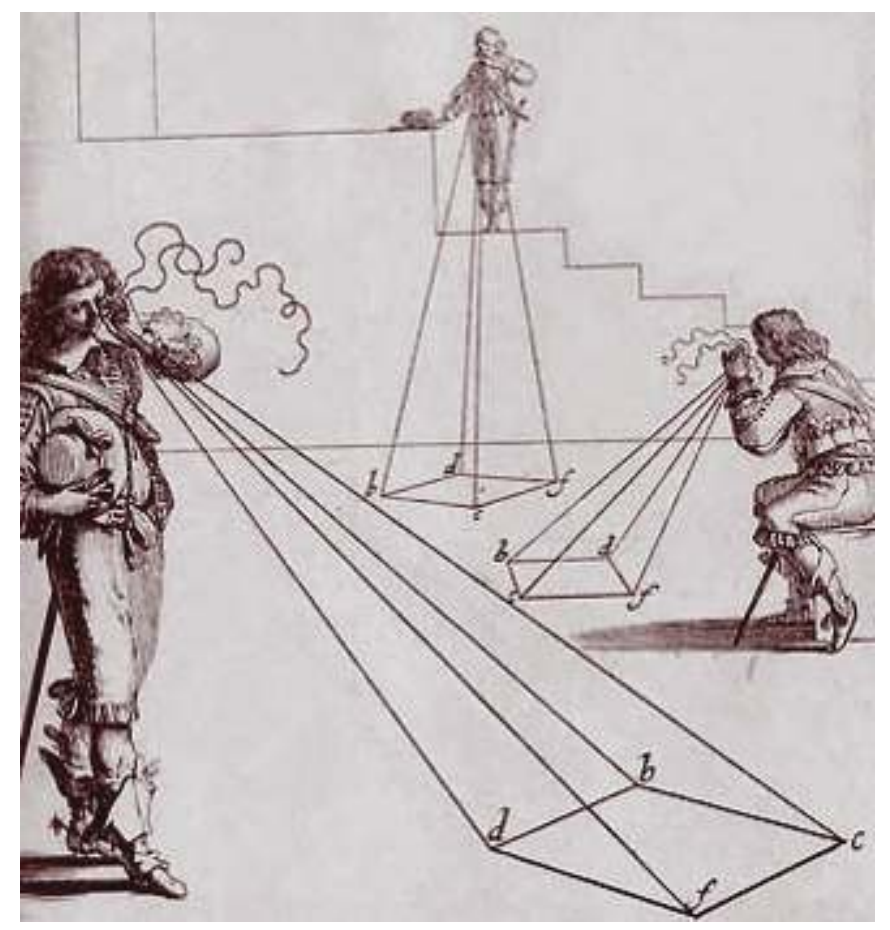

Abraham Bosse, Bildtafel aus: Manière universelle de M. Desargues pour pratiquer la perspective [...], Paris, 1648 (Ausschnitt).

11 Die Form, die ein gespannter Faden annimmt, wenn man ihn loslässt, ist uns paradoxerweise vertraut. Zum Beispiel wird diese Form immer wieder in Abhandlungen über die Perspektive gezeigt; zu didaktischen Zwecken stellt man hier die »optischen Strahlen« als Fäden dar, die an dem Gegenstand befestigt sind, der dargestellt werden soll. Und diese Fäden lässt man dann zum Auge hin zusammenlaufen, indem man sie fest zwischen die Finger klemmt, um so den Vorgang des Sehens nachzuahmen: Der überschüssige Teil dieser Fäden, der also, der »herausguckt« wie ein Unterrock, kräuselt sich in lockeren Windungen in sich selber nach einem allgemeinen Muster, das zwar an das barocke oder manieristische Alphabet der dekorativen Flecht- und Rankenwerke erinnert, aber letztlich doch der wiedergegebenen Realität treu bleibt: der Realität eines sich selbst überlassenen Fadens, wie der der Stoppagen. Solch eine "optische Strahlung « findet sich in Abraham Bosses Illustrationen zur Manière universelle de M. Desargues pour pratiquer la perspective [...] von 1648 oder auch im Thaumaturgus Opticus von Jean-François Niceron (1646). Ihre Verwandtschaft mit Tausenden dekorativer Verfahren verleiht diesen Bildtafeln, die ja a priori demonstrative Ziele verfolgen, etwas von zweckfreier Verzierung, verpackt die Demonstration hübsch in ein Kräuselband, das sie luftiger und anmutiger erscheinen lässt, ohne auch nur für eine Sekunde ihre Zugehörigkeit zur Logik solcher Demonstrationen aufzugeben. So integrieren solche Dekorationen die bei Zeichnungen übliche Sehgewohnheit von Rahmen, Rand oder Bordüre (Parerga, die in manchen Illustrationen von Bosses Traktat auffallenderweise gerade fehlen: Die Abbildungen gehen in der Seite auf, ohne Einrahmung oder Umrandung). Die Arbeiten von Jean Clair haben ausreichend verdeutlicht, inwieweit die Perspecteure in Duchamps Werk herumgeistern ${ }^{23}$, so dass es unnötig ist, hierauf ausführlich zurückzukommenerinnern wir bloß daran, dass eine Notiz in Im Infinitiv ${ }^{24}$ wörtlich sagt: "Siehe Katalog Bibliothek Sainte-Geneviève die ganze Rubrik Perspektive: Niceron (Vater, J.Fr.) Thaumaturgus Opticus«. Ohne jeden Zweifel können diese erstaunlichen Bilder dem 
Künstler zum Zeitpunkt der Stoppagen (1914) in Erinnerung gewesen sein. Doch lassen wir für einen Augenblick die Frage beiseite, ob letztere von Duchamp (im Wortsinn) manipuliert wurden oder nicht: nicht weil diese Frage uninteressant wäre, sondern weil wir uns, bevor wir darauf zurückkommen, für eine Weile den endlosen Assoziationen überlassen wollen, die das so wunderbar rätselhafte Werk von Duchamp wie kein anderes auszulösen vermag. (Interpretationsexzesse sieht dieses Werk übrigens vor und verspottet sie von vornherein - man könnte fast sagen: Es beinhaltet sie).

\section{Die Schreibweise des Unwägbaren ${ }^{25}$}

Denn die zierlichen Spiralen im Faden der Perspekteure rufen ihrerseits andere Bilder, aus anderen Zeiten, ins Gedächtnis: Geheimnisvolle wie jenes, um damit zu beginnen, das dem Chagrinleder als Motto vorangestellt ist (Motto oder Parergon, wir befinden uns immer noch außerhalb des Werks). Balzac, so viel wissen wir, hat bei Laurence Sterne jenen Linienzug ausgeliehen, der in den verschiedenen Ausgaben der Novelle übrigens nach und nach einige Verzerrungen erlitt: So verweist ein Druckfehler den Leser gleich zu Beginn (absichtlich?) auf Kapitel CCCXXII von Tristram Shandy, während die Zeichnung tatsächlich in Kapitel CCCXII erscheint; bei Sterne ist die Linie ziemlich dünn, neigt sich um fünfundvierzig Grad zur Achse der Seite und beschreibt an ihrem rechten Ende eine Drehung um sich selbst: Bei Balzac ist sie spiegelverkehrt, zur Horizontalen gebogen, dicker und ohne Schleife, die sich, entsprechend ihrer Umkehrung, an ihrem linken Ende hätte befinden müssen. Warum Balzac diese Linie an den Beginn des Chagrinleders, jenes orientalischen Pseudomärchens, gestellt hat, bleibt offen. Wollte er, wie meist behauptet wird, mit dieser Form die Unwägbarkeiten des Lebens darstellen? Doch ist die Novelle keine Allegorie des Glückspiels: Der schrumpfende Talisman verkörpert im Gegenteil eine Existenz, die ausschließlich von der Pendelbewegung des ausweglosen Schwankens zwischen Wunsch und Leben bestimmt wird.

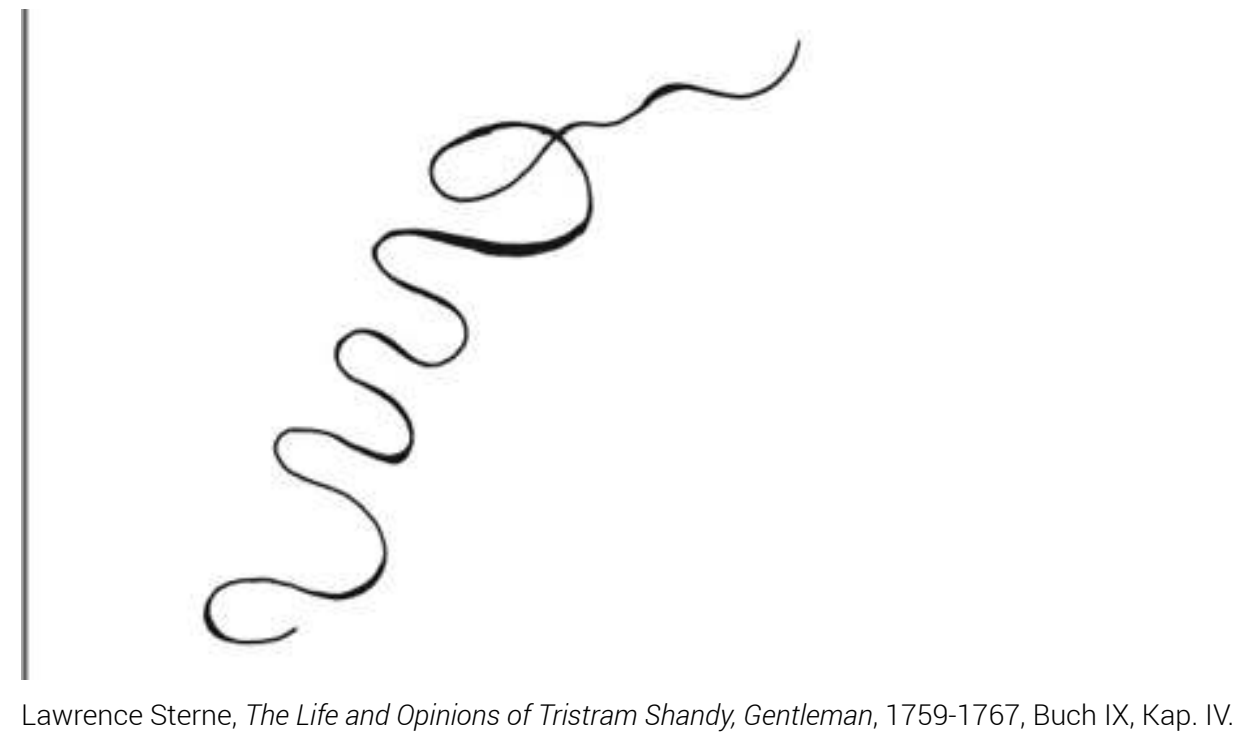




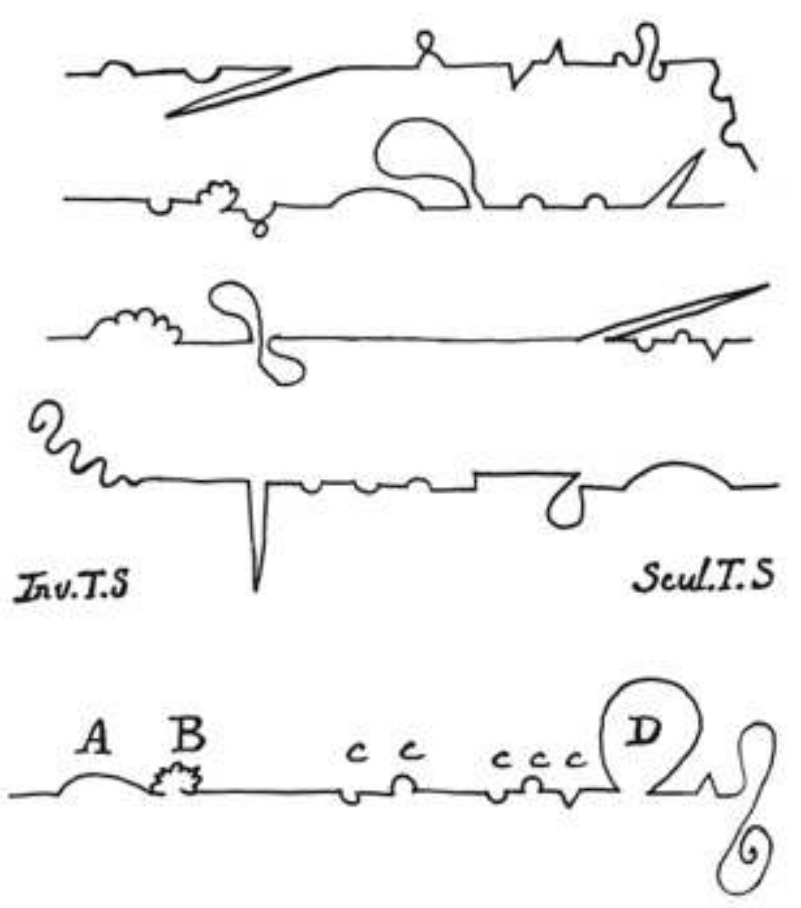

Lawrence Sterne, The Life and Opinions of Tristram Shandy, Gentleman, 1759-1767, Buch VI, Kap. XL. desjenigen nämlich, den der Korporal Trim Unendlichkeit der Perspektiven darstellen will, die sich einem Mann vor der Heirat bieten. »Tausend von meines Vaters scharfsinnigsten Schlussfolgerungen hätten nicht mehr zugunsten der Ehelosigkeit sprechen können«, fügt im Roman Tristram shandy hinzu. ${ }^{26}$ Doch einige Kapitel zuvor finden sich weitere Maßnorm-Stoppagen: Sie werden als Entstellungen des Ideals vorgeführt, das die Gerade ebenso für das Leben (der Moralapostel, der Landvermesser und der »Kohlpflanzer«, sagt der Erzähler) wie für den autobiografischen Bericht darstellt, den Tristram Shandy abgibt: Vier gekrümmte Linien beschreiben schematisch den Gang der Ereignisse, über die in den ersten vier Bänden des Romans berichtet wird. ${ }^{27}$ Eine fünfte Linie, die der Geraden ähnlicher sein soll - was offenbar nicht ins Auge fällt -, wird näher kommentiert: Jede Ausbuchtung, jedes Mäandern wird haarscharf mit einer der "Aus- und Überschreitungen« des Helden in Verbindung gebracht oder auch mit einem Hindernis, auf das er stieß. Daher lässt sich nicht ausschließen, dass die Schlangenlinie im Chagrinleder eine Interpolation aller Linien des Shandy ist, wobei sie jene gebrochenen Linien, welche die Zeit der Erzählung versinnbildlichen - und die sich, wie die Schrift, von links nach rechts entwickeln -, über die luftigere erhebt, in der sich Ehelosigkeit verschlüsselt.

Bei Sterne wie bei Balzac aber beinhalten diese geheimnisvollen Linienzüge den Gedanken, dass eine grafische Darstellung des Unwägbaren möglich sei - werde es nun positiv oder negativ wahrgenommen (ein Junggeselle würde anmerken, dass gerade viele Aspekte des Duchampschen Infrageringen sich dem Unwägbaren nähern: Wenn Duchamp schreibt: »das Mögliche ist ein Infrageringes « ${ }^{28}$, suggeriert er, dass alles, was geschehen kann, infragering ist. Das heißt: unmöglich zu messen; in der Umgangssprache, die das Gewicht dem Metermaß vorzieht, hieße es unmöglich zu wiegen, unwägbar.) 


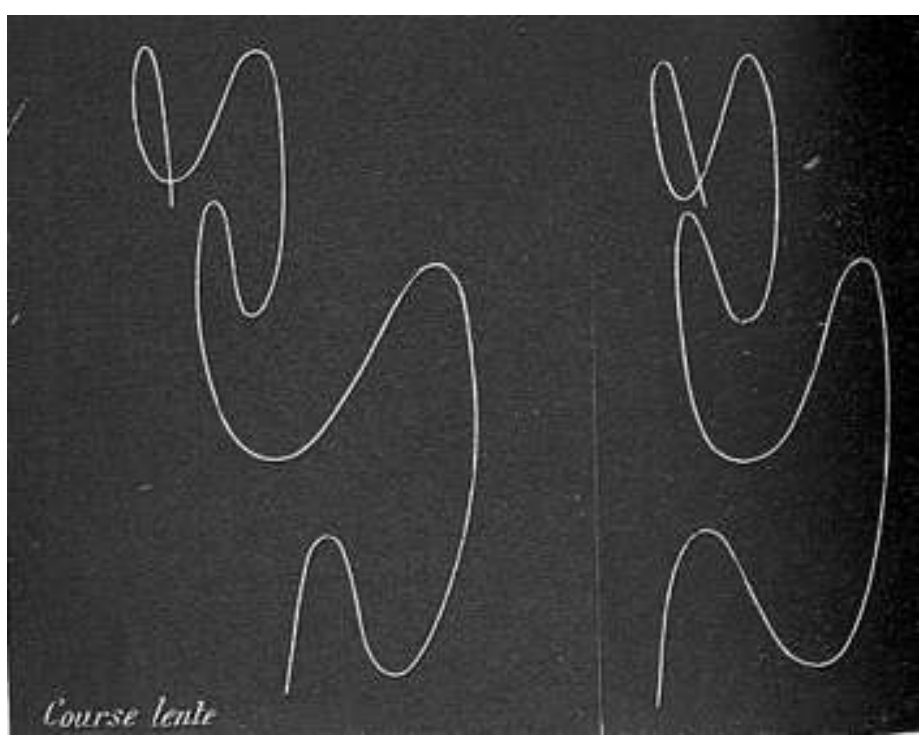

Etienne-Jules Marey, Bewegungsbild: Trajectoire stéréoscopique d'un point brillant placé au niveau des vertèbres lombaires d'un homme qui marche en s'éloignant de l'appareil photographique, in: ders., Le Mouvement, Paris, G. Masson, 1894, Fig. 13.

Ereignisse darstellen, die in die Kategorie des kaum Wahrnehmbaren oder des rein Gedanklichen gehören, Ziffern und Wörter durch die Verstehbarkeit des Zeichens genau da zu ersetzen, wo sie nicht vorstellbar scheint, das ist Sinn und Zweck der grafischen Methode von Étienne-Jules Marey im Jahre 1885. ${ }^{29}$ Ist es ein Zufall, dass die Aufnahmegeräte von Marey ${ }^{30}$ (der Sphygmograf, der Hämodromograf, der Myograf und andere Apparate mit der Endung -graf, deren direkte Nachkommen unsere modernen Krankenhausmonitore sind) Linien produzieren, die den Sterneschen nahe kommen? Tristram Shandy hätte es nicht missfallen, wenn die grafische Wiedergabe seiner Erzählung in etwa das Aussehen eines Elektroenzephalogramms angenommen und seinem Urheber Wehruntauglichkeit attestiert hätte, wenn anders man als Urheber seines eigenen Elektroenzephalogramms bezeichnet werden kann (dies suggerierte ironisch Robert Morris, der im Jahre 1963 seine eigene Hirnstromkurve signierte und ausstellte: Self-Portrait EEG). Eine Hand, die eine Arabeske zeichnet und sich weder zwingt, die Wirklichkeit darzustellen, noch die Buchstaben eines $\mathrm{x}$-beliebigen Alphabets zu formen, bezeugt ihre legitime Zugehörigkeit zu einem imaginären, durch die Fortentwicklung von Wissenschaft und Technik transformierten Kollektiv ganz wie eine Registriernadel, ein Stilett, Seismograf einer tieferen Realität, von deren Existenz und Beschaffenheit sie im selben Augenblick zeugt. Hat das Wort Stil nicht immer mehr die Bedeutung der Zugehörigkeit zu einer Schule verloren, evoziert es nicht jene strikt individuelle, rhythmische Vibration, die im gekonnten Strich einer Zeichnung oder Schrift noch überlebt? Jene kleine Index-Fraktion des Zeichens, die, mit Peirce zu sprechen, am Ikon und am Symbol gerade noch wahrnehmbar ist? Die modernen Handliniendeuter, die Grafologen also, glauben gern, eine geheime Wahrheit in diesem Tanz der Hand aufdecken zu können, der als Tanz eigentlich nur im speziellen Fall der Unterschrift erkennbar ist, in ihrer gewollten oder ungewollten Unleserlichkeit oft der Arabeske nahe. Vermutlich gibt es seit jeher Verbindungen zwischen Kalligrafie und Choreografie. In seiner höchst eigenartigen Abhandlung über normative Ästhetik, der Analyse der Schönheit, siedelt William Hogarth die Linien, die sich aus den Schritten eines Menuett-Tänzers auf dem Fußboden ergeben, unter den gefälligsten an, die man sich vorstellen könne. Mit dem heiklen Problem der grafischen Transkription von 
Tanzschritten konfrontiert, werden viele Künstler natürlich wieder auf die Figuren 122 und 123 in Hogarths Abhandlung stoßen, in denen sich die von der Bewegung der Tänzer erzeugten Linien darstellen: Die Zeichnungen von Sophie Taeuber - einer Freundin Rudolf von Labans und Mary Wigmans und selber eine Tänzerin - oder auch die von Trisha Brown kommen ihnen erstaunlich nahe, und in der Entscheidung, auf dem Bild, das er 1962 bei Sidney Janis auf dem Fußboden ausstellt, einen steifen, schwerfälligen Tanz schematisch darzustellen, zeigt sich die ganze Ironie Andy Warhols.

\section{Die Linie der Schönheit}

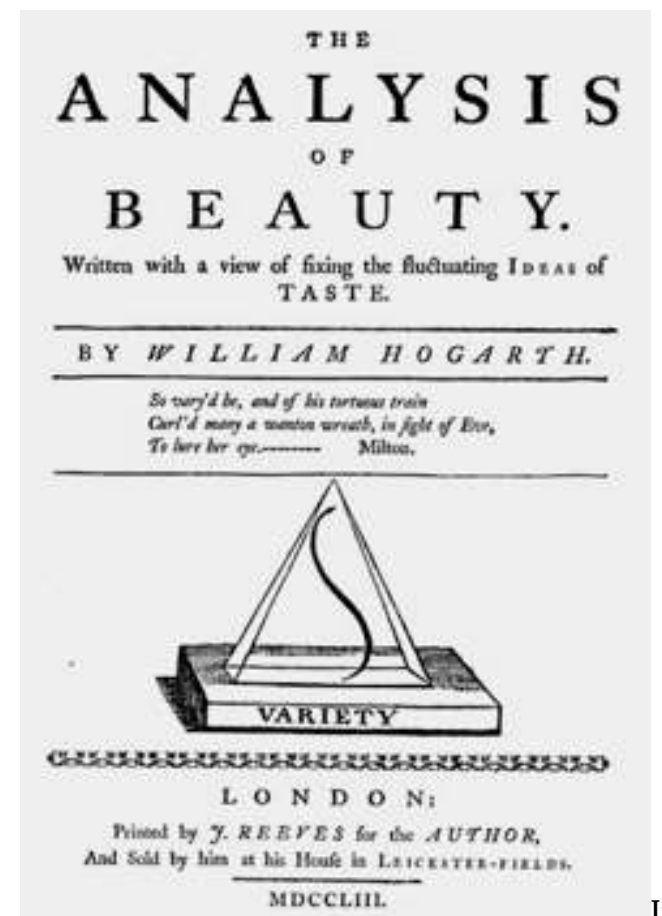
der erste Illustrator des Shandy) können die gefälligen Linien nur gebogen sein oder, genauer gesagt, wellenförmig (waving), wobei der Kreis sich mit der Geraden die untere Stufe der ästhetischen Leiter teilt: Unter den Wellenlinien ist die Linie der Schönheit ${ }^{31}$, die "aus zwei geschwungenen entgegengesetzten Krümmungen besteht«, noch gefälliger, "so sehr, daß die Hand das Lebendige der Bewegung verspürt, wenn sie jene mit Feder oder Pinsel zeichnet«.

William Hogarth, The Analysis of Beauty, London, 1753.

Ebenso wie ihre ältere Schwester, die Linie der Grazie (die man sich veranschaulichen kann, indem man sich einen dünnen Messingdraht vorstellt, »der geschickt um die schöne und mannigfaltige Figur eines Kegels gewunden ist « ${ }^{32}$, gehört die Linie der Schönheit zur Königsriege der spezifischen Nomenklatur der Analyse der Schönheit: der der Schlangenlinien (serpentine lines). Das Adjektiv serpentine ist nicht leichtfertig gewählt. Auf dem Selbstporträt Hogarths (1848-1849) erscheint die Schlangenlinie als Messinstrument, eine Art feines Kaliber (oder Prüfmaß) in der Form eines S, das auf der Palette des Künstlers neben den Farben liegt. Aber auf der Titelseite der Analyse der Schönheit nimmt sie in einem Dreieck die Form eines vergöttlichten Reptils an, 
Markenzeichen des Künstlers und verbildlichtes Milton-Zitat, das als Inschrift erscheint:

So wand sich dieser durch und flocht aus seinem

Gewundnen Schweif gar manche bunte Schleife

Vor Evas Auge, um es herzubannen. ${ }^{33}$

Die Wellenlinie, oder wie immer man diese schwer $\mathrm{zu}$ bezeichnende Figur sonst benennen mag $^{34}$, versteht es, sich jederzeit sowohl mit dem Tierreich wie mit dem der Pflanzen zu verbinden. Die Natur kennt keine Linien. Doch was das Tierreich betrifft, ist es offensichtlich die Schlange, die die wichtigste, äußerste Vergegenständlichung der sich bewegenden Linie bildet, und deren nächstliegendes Äquivalent. Es ist recht logisch, dass gewisse fehlerhafte Ausgaben des Chagrinleders den von Sterne übernommenen Linienzug in eine eindeutig erkennbare Schlange verwandeln. Und dasselbe Tier schlängelt sich durch die Kunstgeschichte, von Laokoon bis zum Schlangenritual der Hopi-Indianer, wo Warburg die Schlange als »ursprüngliche« und rekurrente Formel für Zeit und Bewegung einfängt. ${ }^{35}$

Im Pflanzenreich wimmelt es von Lösungen für das Rätsel der Wellenlinie: Blätter und Blumen sind zur Stelle, um die Linie in ein Rankenwerk zu verwandeln, und die dekorative Arabeske, wie Riegl sie in seinen Stilfragen als »Pflanzenranke der saracenischen Ornamentik « versteht, ist eher ein Blumen- als ein abstraktes Motiv. Die Flora bietet uns ein angenehmeres Bildregister als die Kriechtiere, zu denen die Schlange zählt.

»[...] nichts trägt stärker zum Frieden des Herzens bei, zur Erhebung des Geistes und zu den großen Ideen von Gerechtigkeit und Redlichkeit als das Schauspiel der Wälder und Felder, und die winzigen Partien der Pflanze, die manchmal eine geradezu architektonische Ordnung aufweisen, bestätigen den allgemeinen Eindruck. Kein Sprung, so scheint es, kein falscher Ton, möchte man törichterweise sagen, stört merklich die entschiedene Harmonie der vegetabilischen Natur«,

sagt uns Bataille in einem Artikel aus den Documents, »Die Sprache der Blumen «. ${ }^{36}$ Kein falscher Ton? Bataille räumt ein: „Sicher, tolle Windungen der Klettersprossen, eigenartige Risse im Blattwerk bezeugen, dass nicht alles gleichförmig korrekt ist in der tadellosen Aufrichtung der Gewächse« - so sein Kommentar zu einer ganzseitigen Wiedergabe der packenden Fotografie von Karl Blossfeldt, die die Ranken der Bryona Alba in fünffacher Vergrößerung zeigt (der Laie bezeichnet bryona alba als Teufelsrübe). Das Bescherelle-Wörterbuch definiert die Ranken als »Pflanzenfäden oder Zweige, spiralförmig gewunden, mittels deren es mehreren schwachen Gewächsen gelingt zu klettern, indem sie sich an benachbarte Gegenstände heften«. Eine Definition, die die mit der Schlangenform verbundene Verworfenheit noch der parasitären Funktion jener Pflanzen einschreibt - und wir sollten nicht übersehen, dass in unseren Klimazonen die Weinrebe die am meisten verbreitete Ranke ist, ein schwächliches Gewächs, sündige Räusche verheißend.

Somit entzöge die Ranke sich von Natur aus der besonnenen Komposition dekorativer und stilisierter Pflanzenmotive. Auf den sechs als Schleifen bezeichneten Stichen, virtuosen, von der islamischen Kunst inspirierten Flechtwerken, lässt Dürer am Rande des Hauptmotivs Ranken erscheinen als frei sich bewegende, noch nicht in der prächtigen Lasur des Flechtwerks erstarrte Striche - oder auch so, als wollten diese Ranken sich plötzlich dem Geflecht entziehen und seine unerbittliche Symmetrie zerstören. Und dieser Vibrio scheint im Hintergrund von Dürers Gesamtwerk mitzuschwingen: Im Heiligen Hieronymus im Gehäus von 1514 tritt eine überproportional 
große Ranke, den später von Blossfeldt fotografierten ganz ähnlich, aus einer geheimnisvollen Frucht heraus, einem flaschen- oder birnenförmigen Kürbis. Diese eindeutig allegorische Frucht ließ bei den Humanisten jener Zeit die Kontroverse um eine Stelle aus dem Buch Jona in der Vulgata des heiligen Hieronymus aufkommen: Jonas gelingt es, Ninive mit einer Leichtigkeit zu bekehren, die ihn selber verunsichert, er hält inne und denkt nach; über seinem Kopf lässt Gott alsdann eine Pflanze wachsen, um ihn vor der Sonne zu schützen. War dieses rettende Gewächs bis dahin als cucurbita bezeichnet worden, übersetzt der heilige Hieronymus seinen hebräischen Namen mit hedera, Efeu. Dieser neue Begriff beunruhigte die Gläubigen (weil der Wuchs des Efeus wiederum dem Kriechen einer Schlange ähnlich ist?), und Augustinus machte dies dem heiligen Hieronymus zum Vorwurf: Offensichtlich geht es im Theologenstreit um die Frage von Wortlaut und Sinn, und dieser Disput um die Frucht mit ihren ambivalenten Ranken wird bei Dürer emblematisch. Ebendiese Ranke erscheint 1523 erneut im Großen Triumphwagen Kaiser Maximilians I., hier aber ohne jene Modellierung, die sie im heiligen Hieronymus in die Illusion der Räumlichkeit einbezogen hatte, und zurückgeführt auf ihre grafische Substanz, oder auch zu ihr erhoben. Nach Panofsky ist in diesem für Dürers dekorativen Stil charakteristischen Ensemble »der allegorische Geist auf die Spitze getrieben $\aleph^{37}$ : Die Pferde, die den mit Emblemen aller Art befrachteten Wagen ziehen, passen ihren Schritt dem Wesenszug der personifizierten Tugenden an, die sie leiten: Erfahrung und Geschicklichkeit, Großmut und Kühnheit, Schnelligkeit und Festigkeit ... Oberhalb dieser Grazien, die ihr Gespann mit tänzelnden Schritten führen, schwebt ihr Name. Doch was uns hier vor allem aufmerken lässt, sind die wogenden Ranken, die jeder naturalistischen Bezüge entbehren und ihrerseits in einem unbestimmten Raum schweben - weder so ganz eine Abbildung des Himmels noch bloß das leere Feld der Seite -, und die eine geheimnisvolle Botschaft zu überbringen scheinen. Der Definition nach ist eine Allegorie die Verkörperung einer Abstraktion oder deren Projektion ins Konkrete: Hier sieht es ganz danach aus, als nähme Dürer mit seinen Voluten die paradoxe Herausforderung auf sich, eine abstrakte Allegorie zu schaffen, den letzten Daseinsgrund und die äußerste Gestalt der allegorischen Gattung, eine Allegorie der Allegorie gewissermaßen. Möglicherweise verbirgt sich eine verschlüsselte, aber feststehende Bedeutung hinter den spiralförmigen Arabesken des Triumphwagens: Und wenn sie, betrachtete man Dürer vom Standpunkt der Moderne aus, lediglich eine melancholische Darstellung des nicht Darstellbaren, verführerischer Vibrio der Bilderstürmerei zur Zeit einer die Abgötterei fürchtenden Reformation wären? Sigmar Polke machte dieses Motiv zum zentralen Element seiner Dürerschleifen, die 1986 in Venedig ausgestellt wurden, und brachte damit diese vergessene Karte erneut ins Spiel. 


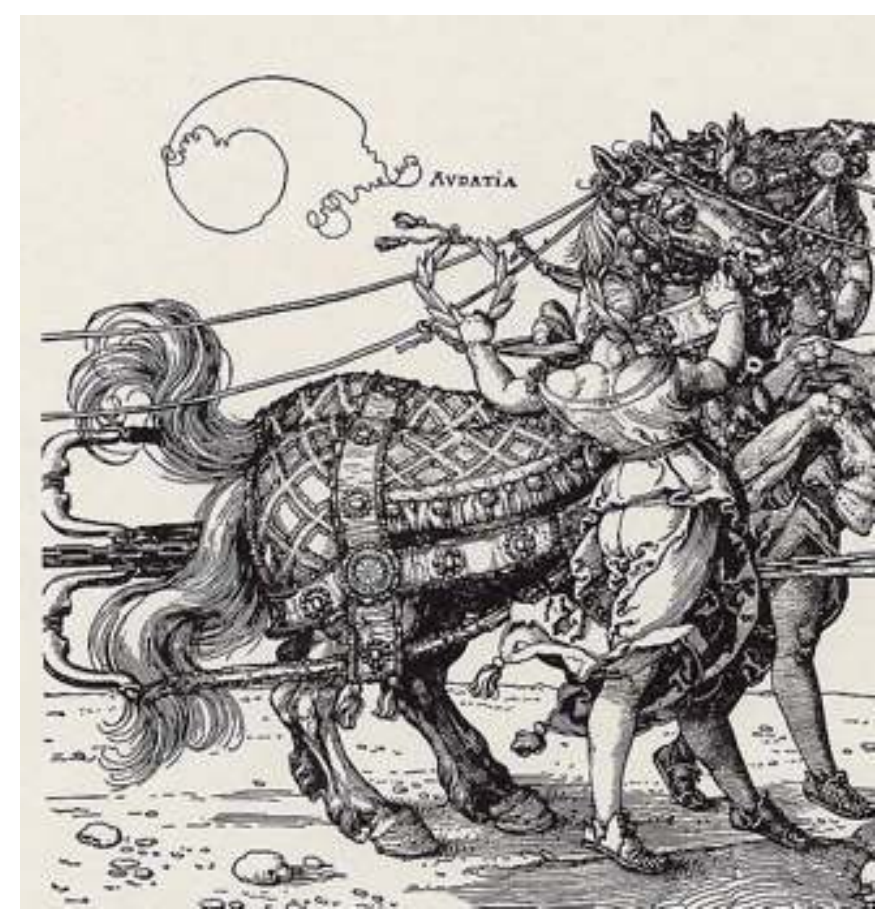

Albrecht Dürer, Triumphzug des Kaisers Maximilian I., 1523, Holzschnitt (Ausschnitt)

\section{Ruhe in der Bewegung}

„Vergessene Karte« ist offenkundig übertrieben. Immerhin durchquert die Wellenlinie zum Beispiel Riegls Stilfragen vom einen Ende zum anderen: Obschon er ihren Stellenwert in der islamischen Kunst ausführlich untersucht, präsentiert er diese Linie in ihrer Gestalt des Rankenwerks als das ganz spezifische Merkmal des griechischen Genius:

»In der mykenischen Kunst begegnet uns überhaupt zum ersten Male eine frei bewegte Pflanzenranke zu dekorativen Zwecken verwendet. Ferner ist die mykenische Kunst, so viel wir sehen können, die Wiege der fortlaufenden sowie der intermittirenden Wellenranke gewesen, d.h. derjenigen zwei Pflanzenrankenmotive, die der griechischen Kunst, und zwar dieser zuerst innerhalb der ganzen antiken Kunstgeschichte, ganz besonders eigenthümlich gewesen sind. ${ }^{38}$

Um aber der Ranke diesen Rang zu erteilen, muss Riegl ihr jegliche naturalistische Herkunft absprechen: Das Pflanzliche ist nur eine Verkleidung der Ranke, nicht ihr Modell:

"Kein Vorbild in der Natur konnte auf das Zustandekommen der Wellenranke unmittelbaren Einfluss üben, da sie sich in ihren beiden typischen Formen, insbesondere in der intermittirenden, in der Natur nirgends findet: sie ist ein frei aus der Phantasie heraus geschaffenes Produkt des griechischen Kunstgeistes. ${ }^{39}$

Worringer, der diese Stelle in Abstraktion und Einfühlung ${ }^{40}$ zitiert, übernimmt von Riegl und von Lipps den Gedanken, dass die Volute als solche Ausdruck des Kunstwollens der Griechen sei und präsentiert sie als die genaue Bruchstelle zwischen den beiden Begriffen, die den Titel seiner Doktorarbeit bilden - der Abstraktion auf der einen, der Einfühlung auf der anderen Seite.

"Die klassische griechische Ornamentik, mit der ägyptischen verglichen, zeigt an Stelle der geometrischen Gesetzmäßigkeit eine organische Gesetzmäßigkeit, deren 
schönstes Ziel Ruhe in der Bewegung ist, lebendiger Rhythmus oder rhythmische Lebendigkeit, in die unser Vitalgefühl mit allem Glück sich versenken kann. Jeder Naturalismus in subalternem Sinne, jedes Abschreiben der Natur fehlt. Wir haben ein reines Ornament auf organischer Grundlage vor uns. Der Unterschied zwischen geometrischer Gesetzmäßigkeit, d.h. solcher, die dem Abstraktionstriebe ihr Dasein verdankt, und organischer Gesetzmäßigkeit, d.h. einer solchen, die sich dem Einfühlungsdrang willig unterordnet, lässt sich am deutlichsten an der Wellenlinie ${ }^{41}$ definieren. Ich nehme das eine Mal eine rein geometrisch konstruierte Wellenlinie, d.h. ich nehme den Zirkel und lasse abwechselnd nach oben oder nach unten geöffnete Halbkreise ineinander übergehen. Einer solchen Wellenlinie vermag unsere Einfühlung nicht ohne Hemmung und Widerspruch zu folgen. [...] Die griechische Wellenlinie dagegen, die sich nie bis zum Halbkreis erweitert und mit dem Zirkel gar nicht zu konstruieren ist, zeigt einen Bewegungsimpuls, den sie in sanftem Schwunge unserem instinktiven Gefühl entsprechend nachgeht. « ${ }^{42}$

21 Danach ist die griechische Volute - weil sie sich wie eine geometrische Kurve ziehen lässt, ohne geometrischen Ursprungs zu sein, und weil sie sich die Bewegung der Natur zueigen macht, ohne sie zu kopieren - das Sinnbild des Gleichgewichts zwischen dem Abstraktionstrieb und dem Beglückungsgefühl in der Vereinigung mit der Weltbewegung. Die Volute unterscheidet sich in der Form nicht so sehr von der Arabeske. Wie die meisten seiner Vorgänger bescheinigt Worringer ihnen eine gemeinsame Genealogie und ein Nebeneinander im Gleichgewicht zwischen Abstraktion und Einfühlung - bis auf einen kleinen Neigungsgrad in der geometrischen Kurve: Die Volute in der griechischen Welt ist offen für die Verlockungen des Naturalismus, die Arabeske eher empfänglich für die Tugenden der Abstraktion. So entbehrt es nicht einer gewissen Logik, wenn zu Beginn des 20.Jahrhunderts die Vorreiter der abstrakten Malerei diese embryonale Form, die beim geringsten Luftzug vom einen in das andere Lager hinüberweht, gewinnbringend zu nutzen wissen. Das Kapitel, das Kandinsky in Punkt und Linie auf Fläche ${ }^{43}$ der gebogenen Linie widmet, scheint Worringers Gedankengang Wort für Wort zu illustrieren. Das geometrische Schema der gebogenen Linie in alternierenden Halbkreisen (Bild 41 des Werks) erzeugt, wenn es aleatorisch positivem oder negativem "Druck" ausgesetzt wird, eine "freiwellenartige Linie«, die um so mehr an Sternes Linie der Ehelosigkeit erinnert, als sie hie und da durch eine »spontane Verdickung« akzentuiert wird (Bild 43 und 44). Der Wechsel von dicken und dünnen Strichen birgt übrigens die Gefahr eines Identitätsverlusts für die Linie in sich, kann doch schon das geringste Übergewicht ins Schleudern bringen, was in den zwei Dimensionen der Fläche Linie blieb: Die Grenze, sagt uns Kandinsky, ist hier ebenso ungewiss wie die Antwort auf die Frage, „wann der Fluß aufhört und das Meer beginnt«, und jene Grenze ergibt sich nur aus der praktischen Erfahrung. Das Bild 49 - „Spontane Nachdrücke einer freien Gebogenen « verleiht dieser Bedrohung Gestalt: Sie liefert uns eine Linie mit Verdauungsstörungen, die in einem entropischen Prozess begriffen ist, der große Ähnlichkeit mit dem des berühmten roten Schlauchs in Jacques Tatis Film Mein Onkel aufweist. Kandinsky verwahrt sich gegen eine allzu große Nähe seiner Bilder mit den Formen der Natur (sie sind, so asketisch sie sich auch ausnehmen, solchen Parallelisierungen eher ausgesetzt als die Rankenwerke, deren naturalistische Wurzeln von Riegl oder Worringer diskutiert werden) und versichert, dass alle Verwandtschaft und Identität sich ausschließlich den Materialien verdanke, die dem Künstler und der ihn umgebenden Welt gemein seien, Linie und Punkt: Die Existenz von schlangenförmigen Pflanzen und von Spinnennetzen, von Kristallen und vibrionartigen Kleinsttierchen bezeuge, dass die Natur sowohl von der geometrisch gebrochenen Linie als auch von der Wellenlinie 
Gebrauch macht. Die Klematisblüten, die Linien, die der Blitz zeichnet, und sogar das »Bindegewebe einer Ratte« liefern ihm weitere Beispiele. Seltsamerweise fehlt unter all diesen naturalistischen Verschwägerungen die Form, die uns den Schlangenlinien Kandinskys heute als die verwandteste erscheint, die des Mäanders. Dieses Erosionsphänomen, dessen wirkliche und gewiss multiple Ursachen noch wenig erforscht sind, würde sich gleichwohl hervorragend als Metapher für den negativen und positiven Druck anbieten, der auf eine Linie ausgeübt wird. ${ }^{44}$ (Aber: die Flussschlinge wird ja üblicherweise nicht zum Familientreffen der Wellenlinien geladen - sei es, weil unsere Wahrnehmung der Mäander inzwischen durch die Verbreitung von Luftaufnahmen geprägt ist, oder aber weil, sicherer noch, sie aufgrund der geologischen Zeit, die sie zu ihrer Entstehung brauchten, schwerfällig und behäbig wirken. Eine Ausnahme, für seine Pariser Liebhaber, bildet hier allerdings das vor ein paar Jahren von der Verkehrsgesellschaft R.A.T.P. in Dienst genommene Logo, eine gelungene Verbindung des Seine-Mäanders mit dem Emblem von Hergés Zigarren des Pharao und ein schöner Kontrapunkt zu der gebrochenen Linie im Logo des Centre Pompidou, zu dem Jean Widmer sich von der Rolltreppe des Gebäudes inspirieren ließ.)

Die Identität der Wellenlinie ist stets in Frage gestellt: Ihre Identität als Linie ist bedroht durch die plötzliche Entstehung einer Fläche, ihre Identität als Abstraktion durch Ähnlichkeiten mit Konkretem, die ihr auflauern. Ihre Zuweisung in das Feld jener Ähnlichkeiten ist ihrerseits ungenau, da sie zwischen Animalischem, Organischem und Pflanzlichem schwankt - und dann wechselt auch noch ihre emotionale Bedeutung, die einmal positiv ist, dann wieder negativ. Mit dem labilen Gleichgewicht, in dem Kandinsky sie hält, bleibt sie indes ein entscheidendes Element in seinem Wortschatz der Abstraktion; besonders präsent ist sie in Gelb-Rot-Blau, seinem großen Bildmanifest von 1925.

In Klees Pädagogischem Skizzenbuch, das zur selben Zeit erschien wie Punkt und Linie zur Fläche, träumt die »aktive Linie, die sich frei ergeht« ${ }^{45}$, (»ein Spaziergang um seiner selbst willen, ohne Ziel« eines Punktes, »der sich verschiebt«) paradoxerweise ${ }^{46}$ von Kultur mehr als von Natur. Spontan nimmt diese Linie eher die Form eines $\mathrm{S}$ wie beim Schallloch der Geige an als die Form einer Schlange oder Ranke, auch wenn der Bezug zu letzterem Motiv in einem kleinen ovalen Ölbild von 1932 ausdrücklich hergestellt wird. Hubert Damisch hat gezeigt ${ }^{47}$, dass Klees Wellenlinie mit dem mathematischen Unendlichkeitssymbol kokettiert, sich im Bild Gleich Unendlich von 1932 bei aller Verschiedenheit sogar als homolog $\mathrm{zu}$ ihm ausgibt (das mathematische Zeichen für "unendlich" ist eine liegende Acht, das heißt die Verschiebung eines Punktes in einem geschlossenen Kreislauf, während die Schlangenlinie eine Öffnung hat). Es scheint ganz, als zögen die unendlich vielen Möglichkeiten, die die Linie in sich trägt, sie bei Klee hin zum Ideogramm, einem weiteren Abgrund, in dem sie sich verlieren könnte, nachdem sie der Fläche, dem Bild der Schlange, des Efeus und des Bindegewebes der Ratte entkommen ist: zum schwindelerregenden Abgrund des kodierten Zeichens, der Schrift, die uns unendlich viele Gedankenkombinationen eröffnet, den Formen aber ihre Sichtbarkeit entzieht, um sie der Domäne des Lesbaren einzugemeinden und damit ihre eigene Gestalt auf den Rang eines Sekundärphänomens zu verweisen. Dieselbe Unsicherheit lässt sich unschwer auch in den Tuschezeichnungen Henri Michaux' feststellen, Katalogen zierlicher Schlangenfiguren, primärer, mühsam an der Grenze zur Schrift sich behauptender Formen, die nur darauf wartet, sie sich einzuverleiben. Und die islamischen Kalligrafien erfinden eine ganze Welt auf jenem äußerst engen 
Raum, der die Wahrnehmung einer Linie als Zeichen von ihrer Wahrnehmung als Dekor trennt.

Ist die Auflistung vermutlich jetzt bereits ermüdend, so wäre die Bestandsaufnahme aller Belege für das Vorkommen der Schlangenlinie im Bereich der abstrakten Kunst und der Avantgarde des 20. Jahrhunderts im allgemeinen nutzlos, wenn sie denn überhaupt möglich wäre. Zwei neuere Beispiele indessen verdienen der Erwähnung. Denn sie werfen erneut die unlösbare Frage auf, die sich bei dieser instabilen Form stellt, das erste Beispiel im Gestus der Ironie, das zweite durch eine subtile Form der Rückkehr zu den Ursprüngen. Es handelt sich um die Walt Disney Productions von Bertrand Lavier (1985) und um die Entrelacs von Hubert Duprat (1992-1994). Wenn Lavier die für Mickeys Abenteuer im Museum für moderne Kunst in den Disney-Studios erzeugten Karikaturen der abstrakten Malerei in den Rang der Bildenden Kunst hebt oder jedenfalls anbietet, sie auf dieses Niveau zu heben -, bescheidet er sich nicht mit dem Effekt einer witzigen Umkehr der Werteskala. Er verdeutlicht damit auch die Treffsicherheit der Karikaturen, die, ohne es zu wissen (vielleicht aber auch gerade wissentlich: Disneys Zeichner sind Künstler), eine mehr oder weniger von Worringer ererbte Aufteilung aufgreifen: zwischen einer regelmäßigen Geometrie einerseits und dem Gewoge der gekrümmten, freiwellenartigen, sich der Einfühlung anbietenden Linie andererseits. Wer in dem Original-Comic ${ }^{48}$ die Parodien abzählt, stellt fest, dass auf beide Kategorien gleich viele entfallen, und zwar fast ausschließlich Streifen auf das geometrische Lager, auf das andere fast ausschließlich Voluten. Von den letzteren hat Lavier mehrere hinreißende Muster festgehalten, deren wohlerzogene Zwillinge sich leicht in Punkt und Linie zur Fläche oder im Pädagogischen Skizzenbuch auffinden ließen.

Die Entrelacs von Duprat wiederum führen ein Paradoxon auf, welches das Schlangenartige ins Lager des Ornaments - im vornehmen Sinne - zurückgeleitet, aus dem es in der Kunst des 20. Jahrhunderts befreit worden war. Ihr Linienzug ist ungezwungen aus freier Hand gezeichnet, nach dem Modell des weiter oben erwähnten Seismografen. Aber dieser Linienzug wird dann sorgfältig abkopiert und mit einem Kupferband als Intarsie auf eine Wand übertragen: A priori ist kein Verfahren vorstellbar, das sich gegenüber den Windungen der Zeichnung widerspenstiger verhalten könnte als diese Art Einlegearbeit aus Gips und Metall. Aus diesem Kontrast jedoch entsteht ein höchst eigenartiges Werk, das die Ambivalenzen der Schlangenlinie in einer Art historischer Sedimentierung verherrlicht, als hätten wir plötzlich eine in Marmor gehauene Vision Pollocks vor uns.

\section{Fabelhafte Mehrdeutigkeit}

Ranke, zu abstrakter Allegorie denaturiert bei Dürer, dann bei Polke, Linie der Ehelosigkeit und Figur unbegrenzter Möglichkeiten bei Sterne, Geheimnis der Grazie bei Hogarth, Chiffre der Abstraktion bei Kandinsky, Schriftwerdung bei Klee, Karikatur bei Disney: der Gedanke, der sich mit der Linie in Bewegung verbindet, ist immer der einer fabelhaften Mehrdeutigkeit, die sie zur offenen Form par excellence macht. Von André Verdet zur Bedeutsamkeit der Arabeske in seinen Wanddekorationen befragt, antwortete Matisse mit der vielleicht besten Definition, die man diesem Motiv unter den Motiven verleihen kann, hat man erst einmal darauf verzichtet, es auf sein göttliches oder dämonisches Wesen als Schlange, Rankenwerk, Kletterwinde - und so fort - $\mathrm{zu}$ reduzieren oder es ans Lächerliche eines heruntergefallenen Fadens zu 
gemahnen: Diese Linie »übersetzt [...] mit einem Zeichen die Gesamtheit der Dinge, sie macht einen einzigen Satz aus allen Sätzen «. ${ }^{49}$ In ihrem, wenn man so sagen darf, ursprünglichen Zustand findet sich die Schlangenlinie bei Matisse nicht allzu häufig: Selbst in den kühnsten, von Ozeanien inspirierten Kollagen verkappt sie sich als Naturelement, als Alge, Blatt oder Muschelschale. Zumindest mussten die Blumen erst die des Bösen sein, um die Arabeske von Matisse aus jedem konkreten Bezug herauszulösen: Sterne hätte sich in den eigenartigen Voluten wiedererkennen können, die der Künstler den Gedichten von Baudelaire hinzufügt. Allerdings bleiben sie auch dann noch marginal gegenüber der Poesie, in die frei gelassenen Stellen am Seitenende gezeichnet wie Ornamente, die das Ende eines Kapitels markieren, und dazu bestimmt, Illustrationen zu ersetzen, deren Drucklegung Matisse derart unzulänglich fand, dass er das Projekt vorübergehend aufgab. Und es ist dieser im doppelten Sinn marginale Bereich, in dem die Schlangenlinie sich enthüllen darf, während sie freilich unterirdisch das Gesamtwerk von Matisse bis hin zu seiner Bildhauerei prägt. Eine kleine Skulptur von 1909 trägt übrigens den Namen Serpentine. Bei Hogarth wird die Linie der Schönheit ihrem Wesen nach als Erzeugerin betrachtet, das heißt als eine Linie, die, im geometrischen Sinne, imstande ist ein Volumen zu »ergeben«, wenn sie sich in drei Dimensionen bewegt. Die "Linie der Grazie« steht am Anfang einer Drehung der »Linie der Schönheit« um eine imaginäre Achse, und diese Bewegung kann, wenn sie fortgesetzt wird, schließlich das Füllhorn als ein exemplarisches Volumen ergeben. So könnte man im berühmten Schlangentanz der Loïe Fuller einen hinreißenden Nachtrag zur Analyse der Schönheit erblicken, der in der Entfaltung der Schleier die Grazie der realen Bewegung mit ihrer flüchtigen Spur zu einer Augenblicksskulptur verbindet.

\section{Die Hypothese eines Arrangements}

Und die Maßnorm-Stoppagen? Rhonda Shearers Hypothese eines Arrangements wird nie mit vollständiger Sicherheit $\mathrm{zu}$ verifizieren sein. Gestützt auf die systematischen Experimente, die sie durchgeführt hat, könnte man jedoch vermuten, das Netz von Kreuzungspunkten, das ein aus der Höhe von einem Meter losgelassener Faden hervorruft, sei so komplex und so umfangreich, dass es nur allzu nachvollziehbar sei, wenn Duchamp das Konfektionsprodukt des Zufalls nachzubessern wünschte: Schließlich näht man ja ohne weiteres einen Saum an einen Anzug, den man »von der Stange " gekauft hat und der nicht genau passt - einen solchen Konfektionsanzug nennt man im Englischen ready-made -, und eine Stoppage bedeutet ja auch so etwas wie eine Ausbesserung. Roger Caillois nahm an, das Walten des Zufalls liege geradezu in der Natur der Dinge in einer Welt, die in ihrem Aufbau unerbittlich auf die endliche Zahl der Elemente aus Mendelejews Periodensystem festgelegt ist. Die Welt der Formen kann sich dem Gesetz nicht entziehen, das die Welt als ganze regiert, Zufälle gibt es hier zuhauf, und dies umso mehr, wenn man es mit einfachen Formen zu tun hat. Sie sind es, deren der Dämon der Analogie sich am leichtesten bemächtigt, und es ist bekannt, dass die Griechen einst die unbehauenen Steine festbanden, die ihre Götter darstellten, aus Angst, sie (die Steine oder die Götter) könnten davonfliegen. Es ist gut vorstellbar, dass Duchamp eine Schlangenlinie, die von der Kunst zur natürlichen Schrift der Welt, der Zeit und der Bewegung, zum Geheimnis von Schönheit und Grazie, zur grafischen Gestalt des Möglichen, ja zur Chiffre der Idee und zur Allegorie der Allegorie geweiht worden war, als mit dem "pataphysischen Zweifel« unvereinbar 
ansah - und wäre diese Linie selbst das Ergebnis einer Zufallsoperation gewesen. Wiederholt erinnert Marcel Duchamp daran, dass die "Indifferenzschönheit" die Frucht von Arbeit ist, selbst wenn diese in Askese bestehen musste: „Die Zahl der readymades pro Jahr begrenzen«, zum Beispiel. Einem allzu großzügigen Zufall - er hat selbstredend seinen Reiz, den aber überlässt Marcel Duchamp dem Surrealismus von Breton - hätte es in der Welt von Duchamp durchaus passieren können, dass ihm eine anti-kosmetische Operation verordnet wird.

Sollte Duchamp sich also, empörend genug, eines anderen besonnen haben? Sollte er statt eines aleatorischen Resultats eine vorteilhafte Darbietung des daran beteiligten Zufalls geliefert haben? Sollten die Maßnorm-Stoppagen eine Allegorie im umfassenden Sinne sein, welche die geflügelten Tugenden eines Puvis de Chavannes einfach durch ein paar schlaffe Fäden ersetzt? Die Hand außer Kurs setzen: dieser Gedanke, zu dem Duchamp sich später bekennen wird, ist in den Notizen zur Schachtel von 1914 jedenfalls noch in keiner Weise erwähnt, und es steht fest, dass diese schriftlichen Anmerkungen als einzige zeitgleich mit den Stoppagen entstanden sind. Fünf der sechzehn Holztafeln beziehen sich mehr oder weniger unstrittig auf den Zufall und die Wahrscheinlichkeitsrechnung (letztere ist Gegenstand eines wichtigen Kapitels in Henri Poincarés La Science et l'hypothèse (1904; dt. Wissenschaft und Hypothese ${ }^{50}$ ), das Duchamp ebenso gelesen hat wie die beiden Ende 1913 oder Anfang 1914 komponierten Partituren des Musical Erratum. Jedenfalls weist alles darauf hin, dass für Duchamp im Jahre 1913 der Zufall und die vierte Dimension mögliche Bewerber um den Posten von Themen der Malerei, von anzustrebenden Zielen darstellen, anstelle des nicht mehr sehr glaubwürdigen Ideals der Symbolisten - das er allerdings der als dumm erachteten Natur der Impressionisten noch vorzog. Daher spricht nichts dagegen, dass in den Stoppagen der Zufall das Thema ist und nicht der Urheber. Seit wann überlässt man denn einem Ideal, welches es auch sei, die niedere Arbeit der Herstellung?

In Kapitel VIII von Huysmans Gegen den Strich versuchte Des Esseintes das, was ihn an den Blumen angezogen hatte, mit seiner ungeteilten Verehrung für das Künstliche zu versöhnen (der Dandy verabscheut die Natur). Nachdem er von der Leidenschaft für künstliche Blumen ergriffen war, musste er sich eingestehen, dass sie alle, sogar die edelsten unter ihnen, der Natur als ihrem Ideal genauestens unterworfen blieben: dass sie, das war der Beweis, die Natur nachäfften. So machte er sich auf die Suche nach "natürlichen Blumen, die falsche nachahmen", die seinen Sieg über die Physis vollenden sollten, indem sie definitiv die Rollen tauschten: Nun war es die Natur, der die unterwürfige Aufgabe der Nachahmung zufiel, und jetzt war es an den industriellen Erzeugnissen, den Ruhm zu ernten und für diese Natur Modell zu stehen. Er sammelte diese Gewächse, und

»sein Ziel war erreicht; kein einziges sah echt aus; Stoff, Papier, Porzellan und Metall schien der Mensch der Natur geliehen zu haben, damit sie ihre Ungeheuer schaffen konnte. Wo es ihr nicht gelungen war, Menschenwerk nachzuahmen, war sie auf das Kopieren der Membranen von Tieren verfallen, borgte sie sich die lebhaften Färbungen ihres verwesenden Fleisches, die herrlichen Scheußlichkeiten ihrer Fäulnis aus. «1

Wir wissen, was Duchamps Positur der Figur des Dandys im allgemeinen und was sie Des Esseintes im besonderen verdankt. Wenn Duchamp die Stoppagen arrangiert haben sollte, dann hat er nichts anderes getan, als mutatis mutandis das Vorgehen von Des Esseintes zu wiederholen: Dadurch, dass man den Zufall nachahmt, wird man ihn nicht entwerten, sondern ganz im Gegenteil ihn ehren. Vielleicht sind die Maßnorm-Stoppagen 
ja wirklich eine Allegorie des Zufalls oder, besser gesagt, sein Porträt oder, sicherer noch, seine Karikatur, denn gewiss ist Einsicht in die Welt für Duchamp nur im Modus der "Bejahungsironie « ${ }^{22}$ denkbar. Die Hypothese eines Arrangements würde seinen nachfolgenden Arbeiten nicht widersprechen: Sind viele der in Arturo Schwarz' Katalog (1964) aufgeführten Werke (In Advance of The Broken Arm, Le porte-bouteilles, Fountain, Pliant de voyage, Trébuchet, Porte-Chapeaux) nicht in Wirklichkeit Repliken von Gegenständen, die anhand von Fotografien entstanden, die ihrerseits in einigen Fällen Fotografien von Repliken waren? Minuziöse Meisterwerke realistischer Skulptur, die voller Eifer die Erzeugnisse einer trivialen Industrie nachbilden? Das Museum, das vor der Weltausstellung auf die Knie fällt? Vielleicht ist der Zufall der Stoppagen ebensowenig aleatorisch wie die ready-mades tatsächlich vorgefertigt sind: Und diese haben mit den Blumen aus Gegen den Strich sicherlich mehr zu tun als mit Duchamps Strategie der Provokation, auf die sie mitunter reduziert werden. Auch wenn das eine das andere nicht ausschließt - vor allem nicht in Duchamps Normandie, wo die Replik "vielleicht - vielleicht auch nicht « mehr als geläufig ist.

Dieser Artikel ist eines Abends bei einem Tischgespräch aus den Anregungen von Ming Tiampo und Georges Didi-Huberman hervorgegangen, viele Hinweise verdanke ich der enzyklopädischen Bildung von Philippe Comar.

\section{BIBLIOGRAPHIE}

Bataille, Georges, »Die Sprache der Blumen«, übers. von Gerd Bergfleth, in: Lessing, Theodor, Blumen, Berlin, Matthes \& Seitz, 2004.

Bonk, Enke, Marcel Duchamp: The Portable Museum, London, Thames \& Hudson, 1989.

Cabanne, Pierre, Gespräche mit Marcel Duchamp, Köln, Verlag Galerie der Spiegel, 1972.

Clair, Jean, „Duchamp et la tradition des perspecteurs «, in: ders., L'Euvre de Marcel Duchamp, Band III: Abécédaire, Paris, Éd. du Centre Georges Pompidou, 1977.

Clair, Jean, Sur Marcel Duchamp et la fin de l'art, Paris, Gallimard, 2000.

Damisch, Hubert, »Égale infini«, in: Critique, n 315-316, August-September 1973, S. 691-723. Auch in: ders., Fenêtre jaune: cadmium ou les dessous de la peinture, Paris, Seuil, 1984, S. 189-228.

Didi-Huberman, Georges, „Sismographies du temps. Warburg, Burckhardt, Nietzsche«, in: Les Cahiers du Musée national d'art moderne, nº 67, 1999, S. 5-20.

Didi-Huberman, Georges, L'Image survivante, Paris, Minuit, 2002.

Duchamp, Marcel, Duchamp du signe. Écrits, zusammengestellt und eingeleitet von M. Sanouillet, Paris, Flammarion, 1975.

Duchamp, Marcel, »Hinsichtlich meiner selbst « (À propos of Myself), in: ders., Die Schriften, Bd. 1, herausgegeben von Serge Stauffer, gestaltet von Peter Zimmermann, Zürich, Regenbogen-Verlag, 1981. 
Duchamp, Marcel, »Peinture de précision et beauté d'indifférence«, in: ders., Notes, vorgestellt von Paul Matisse und Pontus Hulten, Paris, Flammarion, 1999.

Epstein, Jean, Photogénie de l'impondérable, Paris, Éd. Corymbe, 1935.

Hogarth, William, Analyse der Schönheit (1753), übers. von Jörg Heininger, Dresden / Basel, Verlag der Kunst, 1995.

Huysmans, Joris-Karl, Gegen den Strich, aus dem Französischen von Brigitta Restorff, Nachwort von Ulla Momm, Düsseldorf, Patmos / Artemis \& Winkler, 2008.

Journal de Mickey, Neue Folge, Nr. 1279, 2. Januar 1977.

Kandinsky, Wassily, Punkt und Linie auf Fläche. Beitrag zur Analyse der malerischen Elemente, BernBümplitz, Benteli, 7. Aufl. 1973.

Klee, Paul, Pädagogisches Skizzenbuch (1925), Berlin, Gebr. Mann, 1997.

Kostelanetz, Richard, John Cage im Gespräch. Zu Musik, Kunst und geistigen Fragen unserer Zeit, Köln, DuMont, 1989.

Lebensztejn, Jean-Claude, L'Art de la tache. Introduction à la »Nouvelle Méthode« d'Alexander Cozens, o.O., Éditions du Limon, 1990.

Marey, Étienne-Jules, Le Développement de la méthode graphique par la photographie, Paris, Masson, 1885.

Matisse, Henri, Über Kunst, hrsg. von Jack D. Flam, übers. von Elisabeth Hammer-Kraft, Zürich, Diogenes, 1982.

Michaud, Philippe-Alain, Aby Warburg et l'image en mouvement, Paris, Macula, 1998.

Milton, John, Das verlorene Paradies, übers. und hrsg. von Hans Heinrich Meier, Stuttgart, Philipp Reclam jun., 1994.

Panofsky, Erwin, Das Leben und die Kunst Albrecht Dürers, übers. von Lise Lotte Möller, Hamburg, Rogner \& Bernhard, 2. Aufl. 1995.

Poincaré, Henri, Wissenschaft und Hypothese, autorisierte dt. Ausg. mit Erl. und Anm. von F. und L. Lindemann, Berlin, Xenomos, 2003 (Unveränd. Neuaufl. der 4. Aufl. 1928 von B. G. Teubner, Berlin und Leipzig).

Riegl, Alois, Stilfragen. Grundlegungen zu einer Geschichte der Ornamentik, Nachwort von Otto Pächt, München, Mäander, 1985 (Nachdruck der Ausgabe Berlin 1893).

Rousseau, Pascal, »Figures de déplacement, l'écriture du corps en mouvement «, in: Exposé, n², 1995, S. 86-97.

Roussel, Raymond, Eindrücke aus Afrika, Übersetzung von Cajetan Freund, München, Matthes \& Seitz, 1980.

Sartre, Jean-Paul, Der Ekel, aus dem Französischen übertragen von Uli Aumüller, Reinbek bei Hamburg, Rowohlt Taschenbuch Verlag, 1998.

Schwarz, Arturo, The Complete Works of Marcel Duchamp [kommentierter Katalog], New York, Delano Greenidge, 1997; New York, Rizzoli; New York / London, Thames \& Hudson, 1989.

Shearer, Rhonda Roland / Gould, Stephen Jay, Hidden in Plain Sight. Duchamp's 3 Standard Stoppages. More Truly a »Stoppage (An Invisible Mending) Than We Ever Realized, auf der Webseite: tout-fait, the Marcel Duchamp Studies online journal, vol. 1, issue 1, Dezember 1999, Rubrik »News«. 
Steiner, Theo, Duchamps Experiment. Zwischen Wissenschaft und Kunst, München, Wilhelm Fink, 2006.

Sterne, Laurence, Das Leben und die Ansichten Tristram Shandys, übers. von Rudolf Kaßner, Leipzig, Paul List, 1952.

Stevens, Peter S., Formen in der Natur, München / Wien, Oldenbourg, 2. Aufl. 1988.

Worringer, Wilhelm, Abstraktion und Einfühlung. Ein Beitrag zur Stilpsychologie, (1908), hrsg. von Helga Grobing, München, Wilhelm Fink, 2007.

\section{NOTES}

1. Raymond Roussel, Eindrücke aus Afrika, Übersetzung von Cajetan Freund, München, Matthes \& Seitz, 1980, S. 197.

2. Der Logik der Eindrücke entsprechend wird zuerst - unter dem Titel »Händel, sein VesperOratorium mechanisch komponierend « (Roussel [1980], S. 69) - über die Pantomime berichtet; die Anekdote, die hierzu den Schlüssel liefert, wird erst später erzählt - und dieser Schlüssel ist selbstverständlich kaum weniger rätselhaft als das Rätsel, das er zu lösen vorgibt.

3. Siehe Jean-Claude Lebensztejn, L'Art de la tache. Introduction à la »Nouvelle Méthode« d'Alexander Cozens, o.O., Éditions du Limon, 1990, S. 136-139.

4. Nummern 256 und 281 im Schwarz-Katolog (Arturo Schwarz, The Complete Works of Marcel Duchamp [kommentierter Katalog], New York, Delano Greenidge, 1997; New York, Rizzoli; New York / London, Thames \& Hudson, 1989.)

5. Des »Zither spielenden Regenwurms« [A.d.Ü.]. "[...] Auf der Bühne war nur eine Schaufensterpuppe und eine Schlange, die sich ganz langsam bewegte; das war der absolute Gipfel des Ungewöhnlichen« (Pierre Cabanne, Gespräche mit Marcel Duchamp, Köln, Verlag Galerie der Spiegel, 1972, S. 41).

6. 1960 in Tokio.

7. Siehe Schwarz (1997), S. 593.

8. Siehe Lebensztejn (1990), S. 139.

9. 1984 als Live-Sendung aus dem Centre Pompidou übertragen. Von dieser Direktübertragung gibt es mehrere von Paik angefertigte Videofassungen.

10. Vgl. Richard Kostelanetz, John Cage im Gespräch. Zu Musik, Kunst und geistigen Fragen unserer Zeit, Köln, DuMont, 1989.

11. »Note de la boîte verte«. Siehe Marcel Duchamp, Duchamp du signe. Écrits, zusammengestellt und eingeleitet von M. Sanouillet, Paris, Flammarion, 1975, S. 50.

12. "Hinsichtlich meiner selbst« (À propos of Myself), Marcel Duchamp, Die Schriften, Band 1, herausgegeben von Serge Stauffer, gestaltet von Peter Zimmermann, Zürich, Regenbogen-Verlag, 1981, S. 247.

13. Anny zu Roquentin: »Natürlich brauche ich dich nicht zu sehen, wenn es das ist, was du meinst. Weiß du, du hast nichts besonders Erfreuliches für die Augen. Ich brauche, daß du existierst und daß du dich nicht änderst. Du bist wie dieser Meter aus Platin, den man irgendwo in Paris oder Umgebung aufbewahrt. Ich glaube nicht, daß jemals jemand Lust gehabt hat, ihn zu sehen. « »Da täuschst du dich.« »Na ja, ist ja egal, ich nicht. Jedenfalls bin ich froh, zu wissen, daß er existiert, daß er genau den zehnmillionensten Teil des Viertels des Erdmeridians mißt. Ich denke jedesmal daran, wenn man eine Wohnung ausmißt oder wenn man mir Stoff am laufenden Meter verkauft.« (Jean-Paul Sartre, Der Ekel, Aus dem Französischen übertragen von Uli Aumüller, Reinbek bei Hamburg, Rowohlt Taschenbuch Verlag, 1998, S. 217). 
14. Die handschriftliche Anmerkung zur Schachtel von 1914 ist wortgetreu im Katalog von A. Schwarz wiedergegeben (Schwarz [1997], S.335). Die Übertragung des Duchamp du signe (Duchamp [1975], S. 36) fügt ihm zwei weitere Anmerkungen hinzu, die sich hiervon konkret unterscheiden.

15. Siehe Schwarz (1997), S. 594-596.

16. "A propos of Myself«, in: Duchamp (1975), S. 224-225.

17. Schwarz (1997), S. 596.

18. Siehe Enke Bonk, Marcel Duchamp: The Portable Museum, London, Thames \& Hudson, 1989, S. 219.

19. Ein detailliertes Protokoll dieses Experiments findet sich in: Rhonda Roland Shearer und Stephen Jay Gould, Hidden in Plain Sight. Duchamp's 3 Standard Stoppages. More Truly a "Stoppage» (An Invisible Mending) Than We Ever Realized, auf der Webseite: tout-fait, the Marcel Duchamp Studies online journal, vol.1, issue 1, Dezember 1999, Rubrik »News«. Seit Jahren untersucht Rhonda Shearer Marcel Duchamps Werke mit jener hartnäckigen Genauigkeit, die man für gewöhnlich Scotland Yard zuschreibt und an der es die zeitgenössische Kritik - der Autor dieser Zeilen vor allen anderen - törichterweise allzu häufig fehlen lässt.

20. Siehe nochmals Rhonda Shearer: "Doch Duchamp blieb vehement dabei, und sogar dann, wenn er (vielleicht unter dem Eindruck solcher Verdächtigungen) streng befragt wurde. Er blieb dabei, dass er das angegebene Protokoll befolgt habe, indem er jeden Faden - von rexakt Meter Länge - genau ein einziges Mal losgelassen und ihn dort festgeklebt habe, wo er >nach seinem Belieben` gelandet war.« So zum Beispiel in einem Gespräch mit dem jungen Carrol Janis (der uns bedeutete, dass er Duchamp in diesem Punkt festgenagelt habe, weil er diesbezüglich ähnliche Zweifel hegte und ein Unbehagen verspürte). »Marcel: Das konnte man nur einmal machen. Und ich bin auch froh darüber, dass man es nur einmal machen konnte und nicht öfter. Das ist ungefähr so was wie ein Experiment. Das hat mir sehr gefallen ... Carroll: Zu den Fäden wollte ich Sie fragen: Wurden sie nach den Gesetzen des Zufalls losgelassen und in der ersten Position, in die sie gefallen waren, fixiert? Anders gesagt war es demnach so, dass der Faden nur ein einziges Mal heruntergefallen ist? Da gab es nicht mehrere Versuche, bis Sie das Gefühl hatten, dass sich der erwünschte Effekt eingestellt hatte? Marcel: Nein, ich habe dreimal einen Faden fallengelassen. Carroll: Ja, ich weiß, Sie haben unabhängig voneinander dreimal losgelassen. Aber das war bei jedem Faden nur ein einziges Mal? Marcel: Völlig richtig. Das ist ja genau der Punkt... Carroll: Marcel, haben Sie jeden dieser Fäden nur ein einziges Mal herunterfallen lassen, oder haben Sie die Aktion fortgesetzt? Marcel: Nur einmal, wirklich nur einmal. Ich kann mich nicht erinnern, dass es dabei irgendeine Panne gab.«

21. Schearer \& Gould (1999): „Der Anfang jedes dieser Fäden liegt auf der Rückseite. Er zieht sich dann durch ein Nadelloch zur Vorderseite des Werks, schlängelt sich für einen Meter über die Vorderseite (produziert dabei den eigentlichen Linienzug der Stoppage), und danach zieht er sich wiederum durch ein Nadelloch auf die Rückseite zurück und breitet sich hier über einige zusätzliche Zentimeter aus (so dass der Faden auf eine viel größere Gesamtlänge kommt als nach Duchamps Behauptung - sexakt « ein Meter - möglich ist!«

22. Marcel Duchamp, „Peinture de précision et beauté d'indifférence«, in: ders., Notes, Paris, Flammarion, 1999, S. 48.

23. Hierzu lässt sich namentlich auf Jean Clair verweisen: "Duchamp et la tradition des perspecteurs«, in: ders., L'Euvre de Marcel Duchamp, Band III: Abécédaire, Éd. du Centre Georges Pompidou, 1977. Neu aufgenommen und erweitert findet sich der Artikel im »Thaumaturgus opticus", in: ders., Sur Marcel Duchamp et la fin de l'art, Paris, Gallimard, 2000, S. 63-110. Jean Clair rückt die Wellenbewegung des Fadens der Perspecteure nicht in die Nähe der Maßnorm-Stoppagen, sondern in die der "fleischfarbenen Milchstraße« im oberen Teil des Großen Glases: Beweis oder Indiz dafür, dass Marcel Duchamp Die Braut aus den Maschenwerken der klassischen Perspektive 
befreien wollte. Dabei hat er nicht auf diese verzichtet, sondern sich gewissermaßen jenseits des Netzes der gespannten Fäden eingeordnet, aus denen sie sich aufbaut.

24. Duchamp (1975), S. 122.

25. Jean Epstein gewidmet, dem Verfasser des 1935 erschienenen Photogénie de l'impondérable (Paris, Éd. Corymbe).

26. Laurence Sterne, Das Leben und die Ansichten Tristram Shandys, übers. von Rudolf Kaßner, Leipzig, Paul List, 1952, S. 624. Im Originaltext heißt es genau: »Whilst a man is free - cried the Corporal, giving a flourish with his stick thus - A thousand of my father's most subtile syllogisms could not have said more for celibacy.«

27. Zur Ausstellung "Chambres d'amis« von 1986 in Gent (Museum van Hedendaagse Kunst) hatte Luciano Fabro mit Hilfe einer Art Schablone, die diesen Linien entsprach, ein gigantisches Laken zerschnitten (C'est la vie, Baumwollleinwand, variable Dimensionen, Fnac.)

28. Duchamp (1999), Anm. 1, S. 21. Obwohl er schreibt »infragering (adjekt.) nicht Nomen - nie ein Substantiv daraus machen«, gebraucht Marcel Duchamp »infragering « einmal als Substantiv, dann wieder als Adjektiv. Er schreibt die Elemente dieses Kompositums bald aneinander, bald getrennt, bald mit Bindestrich. [Nach Theo Steiner, Duchamps Experiment. Zwischen Wissenschaft und Kunst, München, Wilhelm Fink, 2006, S.236, wäre der Neologismus infra-mince- im Englischen infra-thin - ins Deutsche auch mit unter-gering zu übersetzen; A.d.Ü.]

29. Étienne-Jules Marey, Le Développement de la méthode graphique par la photographie, Paris, Masson, 1885.

30. Siehe zu diesem Thema die Artikel von Pascal Rousseau, (»Figures de déplacement, l'écriture du corps en mouvement «, in: Exposé, $\mathrm{n}^{\circ}$ 2, 1995, s. 86-97), und von Georges Didi-Huberman (»Sismographies du temps. Warburg, Burckhardt, Nietzsche«, in: Les Cahiers du Musée national d'art moderne, $\mathrm{n}^{\circ}$ 67, 1999, S. 5-20).

31. William Hogarth, Analyse der Schönheit (1753), übers. von Jörg Heininger, Dresden / Basel, Verlag der Kunst, 1995, S. 77.

32. Hogarth (1995), S. 79.

33. John Milton, Das verlorene Paradies, übers. und hrsg. von Hans Heinrich Meier, Stuttgart, Philipp Reclam jun., 1994, 9. Buch, S. 268 f. [Die deutsche Ausgabe der Analyse der Schönheit zitiert neben dem im Text erwähnten Selbstporträt von Hogarth auf der Titelseite - als Faksimile der Originalausgabe - Milton im englischen Original: So vary'he, and of his tortuous train/Curl'd many a wanton wreath, in fight of Eve,/To lure her eye. Milton; A.d.Ü.].

34. Einer meiner Freunde wollte sie Zigloogloo nennen, wahrscheinlich eine Kontraktion von Zickzack und looping oder loop: gewunden und geknickt, beides zusammen.

35. Siehe Philippe-Alain Michaud, Aby Warburg et l'image en mouvement, Paris, Macula, 1998, und Georges Didi-Huberman, L'Image survivante, Paris, Minuit, 2002.

36. Georges Bataille, »Die Sprache der Blumen«, Essay übers. von Gerd Bergfleth, abgedruckt in: Lessing, Theodor, Blumen, Berlin, Matthes \& Seitz, 2004, S. 234.

37. Erwin Panofsky, Das Leben und die Kunst Albrecht Dürers, übers. von Lise Lotte Möller, Hamburg, Rogner \& Bernhard, 2. Aufl. 1995, S. 241.

38. Alois Riegl, Stilfragen. Grundlegungen zu einer Geschichte der Ornamentik, Nachwort von Otto Pächt, München, Mäander, 1985 (Nachdruck der Ausgabe Berlin 1893), S. 126.

39. Riegl (1985), S. 127.

40. Wilhelm Worringer, Abstraktion und Einfühlung. Ein Beitrag zur Stilpsychologie, hrsg. von Helga Grobing, München, Wilhelm Fink, 2007, S. 124.

41. Ein Echo auf Hogarths Waving line?

42. Vgl. Worringer (2007), S. 80.

43. Wassily Kandinsky, Punkt und Linie auf Fläche. Beitrag zur Analyse der malerischen Elemente, Bern-Bümplitz, Benteli, 7. Aufl. 1973. 
44. Zu diesem Thema siehe Peter S. Stevens, Formen in der Natur, München / Wien, Oldenbourg, 2. Aufl. 1988. (Für diesen Hinweis danke ich Sylvie Mas.)

45. Paul Klee, Pädagogisches Skizzenbuch (1925), Berlin, Gebr. Mann, 1997, S. 6.

46. Für Klee bleibt die »Zwiesprache mit der Natur [...] conditio sine qua non« (zitiert bei Helene Schmidt-Nonne: »Der Unterricht von Paul Klee in Weimar und Dessau«, in: Klee [1997], S. 54).

47. Hubert Damisch, "Égale infini«, in: Critique, $\mathrm{n}^{\circ}$ 315-316, August-September 1973, S. 691-723. Wieder aufgenommen in ders., Fenêtre jaune: cadmium ou les dessous de la peinture, Paris, Seuil, 1984, S. 189-228.

48. "Traits très abstraits« [»Sehr abstrakte Striche«, das französische Wortspiel lässt sich nicht ins Deutsche übertragen, A.d.Ü.], Journal de Mickey, Neue Folge, Nr. 1279, 2. Januar 1977.

49. Henri Matisse, Über Kunst, hrsg. von Jack D. Flam, übers. von Elisabeth Hammer-Kraft, Zürich, Diogenes, 1982, S. 251.

50. Henri Poincaré, Wissenschaft und Hypothese, autorisierte dt. Ausg. mit Erl. und Anm. von F. und L. Lindemann, Berlin, Xenomos, 2003 (Unveränd. Neuaufl. der 4. Aufl. 1928 von B. G. Teubner, Berlin und Leipzig).

51. Joris-Karl Huysmans, Gegen den Strich, aus dem Französischen von Brigitta Restorff, Nachwort von Ulla Momm, Düsseldorf, Patmos / Artemis \& Winkler, 2008, S. 105.

52. „Die >Bejahungsironie (im Unterschied zur >negierenden Ironie`, die blo $\beta$ vom Lachen abhängt)«, in: Duchamp [1981], S. 174).

\section{INDEX}

Schlüsselwörter : Maßnorm-Stoppagen, Ranke, Schlangenlinie, Stil, Zufallsmusik

Mots-clés : ligne serpentine, musique du hasard, rinceau, Stoppages étalons, style

\section{AUTEURS}

\section{DIDIER SEMIN}

Professor für Kunstgeschichte an der École nationale supérieure des beaux-arts (Ensba), Paris 